\title{
Diagnóstico del patrimonio cultural tangible como visión para el turismo comunitario en el cantón La Maná Provincia de Cotopaxi - Ecuador
}

Diagnosis of tangible cultural heritage as a vision for community tourism in the La Maná canton Cotopaxi Province - Ecuador

Olga Selena Rosero Perez. ${ }^{1}$, Nataly Silvana Yepez Arauz. ${ }^{2}$, Gabriela Isabel Armas Cedeño. ${ }^{3}$ \& Natalia Geoconda Zambrano Cuadro. ${ }^{4}$

Resumen.

DOI: https://doi.org/10.33262/concienciadigital.v3i3.1.1346

Introducción. El diagnóstico del patrimonio cultural tangible como visión pata el turismo comunitario es de suma importancia ya que representa al Cantón conjunto con sus parroquias y comunidades existentes. Uno de los principales objetivos del cantón la Maná y sus habitantes debe ser el rescate de sus costumbres, fiestas, historia, tradiciones, etc. Objetivo. Diseñar una ruta cultural como patrimonio de la humanidad en el cantón La Maná y establecer estrategias de promoción y difusión de la ruta cultural. Metodología. Para el desarrollo de la investigación se utilizó los métodos cuali-cuantitativa, se trabajó con información primaria (levantamiento de campo, entrevistas a informantes claves, talleres), se utilizó el método analítico para el diagnóstico de la situación actual del turismo cultural, sistematizar la oferta y demanda del turista con estas técnicas permitirá aprovechar el potencial turístico. Consecutivamente se llevó acabo el levantamiento del instructivo para fichas del inventario inmaterial basados en la

\footnotetext{
${ }^{1}$ Universidad Técnica de Cotopaxi Extensión La Maná, Ingeniería en Ecoturismo, La Maná - Ecuador., olga.rosero3507@utc.edu.ec

${ }^{2}$ Universidad Técnica de Cotopaxi Extensión La Maná, Ingeniería en Ecoturismo, La Maná - Ecuador., nataly.yepez8013@utc.edu.ec

${ }^{3}$ Universidad Técnica de Cotopaxi Extensión La Maná, Ingeniería en Ecoturismo, La Maná - Ecuador., gabriela.armas0981@utc.edu.ec

${ }^{4}$ Universidad Técnica de Cotopaxi Extensión La Maná, Ingeniería en Eco turismo, La Maná - Ecuador., natalia.zambrano@utc.edu.ec
} 
normativa según el INPC. Resultados. Se logró identificar que el porcentaje más alto es el 48\% que pertenece a la provincia de Cotopaxi, realizando la tabla de los atractivos culturales tangibles, actualmente vigentes en el área turística, dándonos como resultado un aporte positivo al desarrollo socio-económico de la localidad. Conclusión. El inventario de los sitios culturales tangibles del cantón La Maná se encuentra creado por: bienes inmuebles, y manifestaciones religiosas que al juntarse dan un total de quince bienes los mismos que son de tipo artesanal y religiosos estos bienes culturales se encuentran en buenas condiciones físicas para ser promocionados como atractivos turísticos.

Palabras clave: Atractivos, cultura, diagnóstico, difusión, patrimonio, turismo.

\begin{abstract}
.
Introduction. The diagnosis of tangible cultural heritage as a vision for community tourism is of utmost importance since it represents the Canton together with its existing parishes and communities. One of the main objectives of the Maná canton and its inhabitants should be to rescue their customs, festivals, history, traditions, etc. Objective. Design a cultural route as a world heritage site in the La Maná canton and establish promotion and dissemination strategies for the cultural route. Methodology. For the development of the research the qualitativequantitative methods were used, we worked with primary information (field survey, interviews with key informants, workshops), the analytical method was used to diagnose the current situation of cultural tourism, systematize the supply and demand of the tourist with these techniques will allow to take advantage of the tourist potential. Consecutively, the instructions for intangible inventory records based on the regulations according to the INPC were carried out. Results. It was possible to identify that the highest percentage is $48 \%$ belonging to the province of Cotopaxi, making the table of tangible cultural attractions, currently in force in the tourist area, resulting in a positive contribution to the socio-economic development of the town. Conclusion. The inventory of the tangible cultural sites of the La Maná canton is created by: real estate, and religious manifestations that when brought together give a total of fifteen properties, the same that are artisanal and religious, these cultural assets are in good physical condition for be promoted as tourist attractions.
\end{abstract}

Keywords: Attractions, culture, diagnosis, dissemination, heritage, tourism. 


\section{Introducción.}

Ecuador es una nación multiétnica y pluricultural, su población sobrepasa los 16 millones de habitantes. Entre la costa, sierra, amazonia e insular se guardan muchos patrimonios, tanto naturales como culturales, pero en el caso del patrimonio cultural la UNESCO (2009) lo define como el conjunto de obras de los artistas, arquitectos, músicos, escritores y sabios de un pueblo o comunidad. Y el patrimonio cultural tangible se compone de los monumentos, edificios, lugares arqueológicos, conjuntos históricos, etc.

Compartimos con Peña, (2013, p. 32) cuando confirma que "En su evolución histórica la humanidad ha elaborado varios conocimientos para apoyar su desarrollo y su comunicación, los cuales han sido relacionados en diversas manifestaciones y representaciones culturales para ser transmitidos, es fundamental para la identidad y memoria de la sociedad. Los bienes inmuebles que conforman el patrimonio cultural de la humanidad poseen detalles históricos, culturales, folclóricos, literarios, etc. que permiten valorarlas social y patrimonialmente". (Peña, 2013)

(Chasiluisa \& Cupuerán, 2012) Nos comparte que "En el Ecuador existen espacios alternativos para conocer, en los que se trata de establecer una experiencia diferente y una relación cercana con las comunidades. Estos espacios permiten observar las características de la multiculturalidad".

(MINISTERIO DE CULTURA Y PATRIMONIO, s/f) Nos comparte que La cultura de Cotopaxi se caracteriza al igual que otras provincias cercanas por su mestizaje y la profunda fe católica, durante las principales fiestas culturales de la provincia se puede observar esa mezcla armoniosa que convoca a turistas de toda la nación, así como en cada uno de sus cantones (Pujilí, La Maná, Sigchos, Pangua, Salcedo, Saquisilí, Latacunga) por lo cual con el transcurso del tiempo se han convertido en parte del patrimonio cultural tangible.

La provincia de Cotopaxi ofrece varios lugares culturales enfocados en el fortalecimiento de la identidad cultural dándole un valor histórico a los bienes tangible, como visión para un turismo comunitario responsable y así los turistas puedan visitar y convivir con las distintas tradiciones y costumbre que tiene cada comunidad, lo que permite experimentar un contexto diferente, con la variedad de elementos de la naturaleza y las relaciones comunitarias. 
El cantón La Maná cuenta con varias manifestaciones y representaciones que han acompaño a la sociedad en su desarrollo y que son testimonio fiel de su construcción como son los sitios arqueológicos, monumentos, obras de arte, libros, etc. La salvaguarda de estos valores y bienes culturales tangibles como visión turística es esencial para garantizar la trasmisión de costumbres, tradiciones y valores para promover la conexión social de una comunidad con otra. La información en la actualidad sobre atractivos turísticos culturales en el cantón es mínima o desactualizada, poco asociable lo cual afecta la promoción de los destinos turísticos, afluencia turística, debido al desconocimiento de la diversidad atractivos culturales, actividades y servicios turísticos con los que cuenta el cantón ubicación geográfica idónea, clima para poder desarrollar turismo cultural. Es por esta razón que se están buscando rutas alternas para fomentar el crecimiento económico y cultural del cantón, para beneficio de los habitantes del mismo.

Este estudio es una propuesta de ayuda para la promoción del turismo para todas las edades con el afán de proporcionar y dar a conocer nuevas rutas turísticos culturales, teniendo como principal objetivo enfocarse en lograr el desarrollo turístico y su efecto en los turistas para contribuir en el desarrollo económico y social del cantón La Maná y su zona de influencia. El Cantón cuenta con los principales servicios para ofrecer principalmente hospedaje, alimentación, transporte, etc.

El objetivo del siguiente documento es diagnosticar, diseñar una ruta cultural como patrimonio de la humanidad en el cantón La Maná y establecer estrategias de promoción y difusión de la ruta cultural. Todo ello realizando diferentes actividades como es investigar la cultura histórica del cantón La Maná (festividades, tradiciones, religión, arqueológico, arquitectura, artesanía, museo, gastronómico, música y danza), realizando un análisis FODA, inventario de sitios culturales del cantón para su debido diagnóstico. Para el diseño de la ruta se realizaron actividades como es la selección de los atractivos culturales, georeferenciación y la realización de itinerarios y guiones. Por ultimo diseñar y establecer estrategias de promoción y difusión de la ruta para fortalecer a la identidad cultural del cantón La Maná.

Por medio de la difusión en los medios de comunicación transmisión y documentos, con el objetivo de fortalecer su identidad, permitiendo al turista valorar la formas de vida tradicionales tener conciencia y disfrutar del encanto ancestral ya que el turismo es reconocido como una 
activad importante que beneficia social y económica a las diferentes comunidades locales debido a las ventajas que tiene para integrarse a la economía, también es considerado un importante generador de empleo y una de las primeras industrias del mundo, esto hace que se habrá posibilidades para los prestadores de servicios que permiten potenciar y desarrollar cada uno de estos atractivos.

\section{Metodología}

Para el desarrollo de la investigación se utilizó los métodos cuali-cuantitativa, se trabajó con información primaria (levantamiento de campo, entrevistas a informantes claves ,talleres) con representantes gobierno locales, academia, prestadores de servicios del cantón La Maná invitándolos así a diferentes capacitaciones sobre temas específicos que se relacionan al turismo para buscar soluciones o mejoras el sector turístico cultural, además se investigó en fuentes de información secundaria como (revista, libros, redes sociales, manuales de turismo, artículos académicos, tesis, sitios web como el Ministerio de Turismo, Cámara de Turismo, Instituto Nacional de Patrimonio Cultural, GAD Municipal La Maná, GAD Parroquial de Guasaganda, EMTURCOM-LM). Se levantó fichas de campo y georreferenciación donde se obtuvo información sobre los diferentes atractivos culturales del Cantón la Maná.

Se utilizará el método analítico para el diagnóstico de la situación actual del turismo cultural, sistematizar la oferta y demanda del turista con estas técnicas permitirá aprovechar el potencial turístico que ofrece el cantón La Maná, con criterios de sostenibilidad en el manejo organizado de las personas y con sustentabilidad en el aprovechamiento racional de los recursos culturales tangibles que tiene a su alcance, lo que llevaría a mejorar el nivel de vida de sus habitantes; dando a conocer su herencia patrimonial y cultural.

Para la elaboración e interpretación de la Matriz FODA se realizó varias salidas de campo a los lugares culturales, utilizando el método sintético para interpretar la información recabada a través de las visitas in situ. Consecutivamente se llevó acabo el levantamiento del instructivo para fichas del inventario inmaterial basados en la normativa según el INPC (2011, p. 163-164), ámbito: A4 Conocimientos y usos relacionados con la naturaleza y el universo y, sub-ámbito A4.4 Espacios Simbólico; ámbito: A5 Técnicas artesanales tradicionales y sub-ámbito A5.1 Técnicas Artesanales Tradicionales, se pide llenar los campos de gran utilidad en donde se ubica indagación puntualizada. 
A través del método descriptivo consistió en realizar una descripción del cambio en que se encuentra los bienes culturales tangibles del cantón, en donde se estableció las características principales de los sucesos históricos de cada uno de estos bienes tangibles, de esta manera se obtuvieron datos fundamentales, para plasmarlos en el Inventario Cultural Tangible del cantón La Maná. Se aplicó la fórmula de la muestra para determinar el universo de estudio tomando la población total del cantón La Maná según el INEC (2010) el total de población es de 42.216 habitantes, de acuerdo a cifras proporcionadas por el último Censo de Población y Vivienda 2010.

A través de la técnica de muestreo mediante la fórmula según (García, RedingBernal, \& LópezAlvarenga, 2013):

\section{Donde:}

- $\mathbf{N}=$ Es el total de la población.

- $\quad \mathbf{Z}=1,96$ al cuadrado (si la seguridad es del 95\%).

- $\quad \mathbf{P}=$ Proporción esperada (en este caso 5\%=0.5).

- $\mathbf{Q}=1-\mathrm{p}$ (en este caso $1-0.05=0.95)$.

- $\quad \mathbf{D}=$ Precisión $(5 \%=0.05)$

$$
\begin{gathered}
n=\frac{N * Z^{2} a p * q}{d^{2} *(N-1) Z^{2} a * p * q} \\
n=\frac{42216 * 1.96^{2} * 0.05 * 0.95}{0.05^{2} *(42216-1)+1.96^{2} * 0.05 * 0.95} \\
n=73 \text { Respuesta }
\end{gathered}
$$

Una vez empleada la fórmula de muestreo se obtuvo como resultado un total de 73 encuestas, que fueron aplicadas imparcialmente a turistas locales y nacionales. Se recopilo información mediante el instrumento del cuestionario a los turistas en temporada alta como es en el mes de Diciembre se aplicó 73 encuestas y en el mes de Febrero otras 73 dándonos así un total de 146 encuestas, con el fin de conocer la acogida o la demanda que generara los atractivos culturales dentro del cantón La Maná, ya que la misma reflejo resultados positivos para el desarrollo socioeconómico.

También se utilizó la técnica de observación para verificar el estado de los lugares culturales de manera que se pueda priorizar los sitios vulnerables del cantón para posteriormente plasmarlo en un plan de conservación de territorio. 
Tabla $N^{o}$ 1: Sitios Culturales del cantón La Maná

\begin{tabular}{|c|c|c|c|c|}
\hline $\mathbf{N}^{0}$ & $\begin{array}{l}\text { Datos de } \\
\text { Identificación }\end{array}$ & $\begin{array}{lll}\text { Datos } & \text { de } & \text { Jerarquía } \\
\text { Localización } & & \end{array}$ & Descripción & Fotografía \\
\hline & & $\begin{array}{l}\text { Provincia: } \\
\text { Cantón: } \\
\text { Parroquia: } \\
\text { Coordenadas: }\end{array}$ & & \\
\hline
\end{tabular}

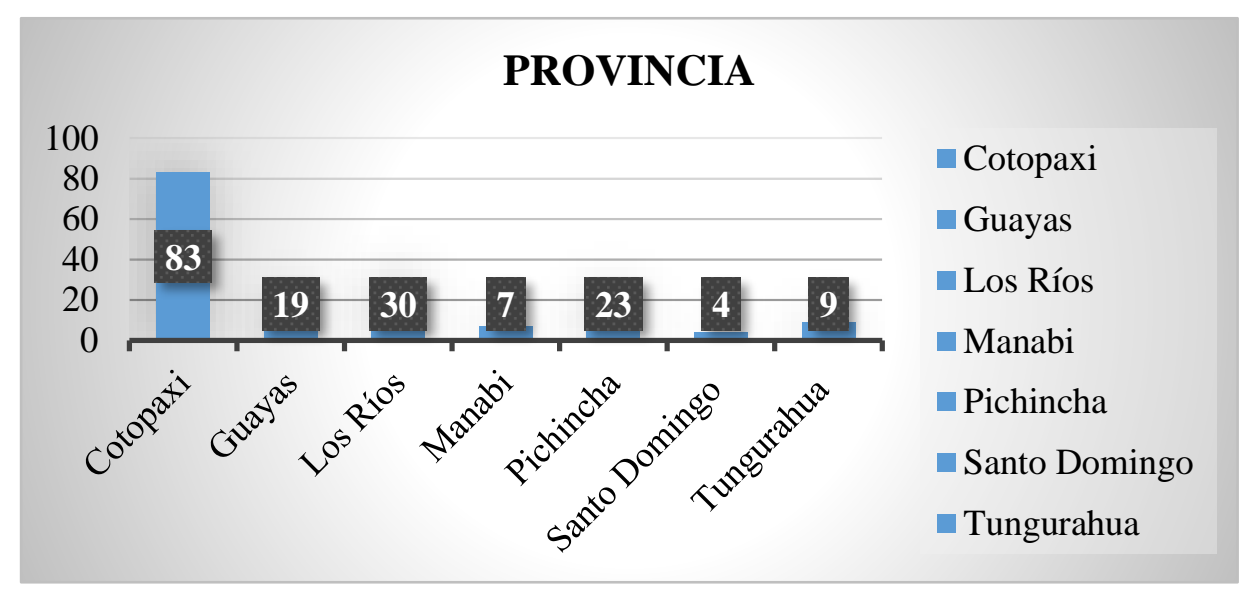

Figura 1: Auto identificación en porcentajes de turistas en el Cantón La Maná Fuente: Olga Rosero et al. 2020.

Análisis 1: Mediante la pregunta del lugar de procedencia, se logró identificar que el mayor porcentaje que es el $48 \%$ pertenecen a la provincia de Cotopaxi, con el $17 \%$ que le corresponde a la provincia de Los Ríos, el 13\% perteneciente a la provincia de Pichincha, $11 \%$ le corresponde a la provincia del Guayas y como porcentajes menores tenemos a las provincia de Tungurahua con el 5\%, a la provincia de Manabí con el 4\% y la provincia de Santo Domingo con el $2 \%$.

\section{Desarrollo socioeconómico comunitario}

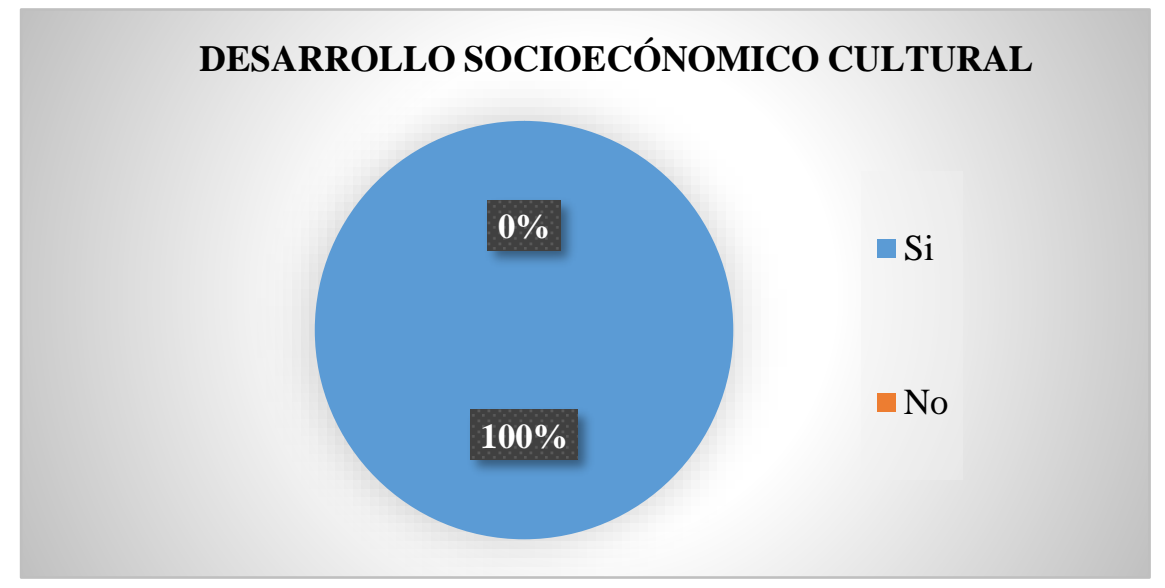

Figura 2: Auto identificación en porcentajes de turistas al aporte socio- 
económico en el cantón.

Fuente: Olga Rosero et al. 2020.

Análisis 2: Correspondiente a la pregunta del desarrollo socio-económico, tenemos el $100 \%$ de aceptación por parte de los turistas y personas de la localidad.

Tabla $N^{\circ}$ 2: Sitios Culturales del cantón La Maná

\begin{tabular}{|c|c|c|c|c|c|}
\hline $\mathbf{N}^{\circ}$ & $\begin{array}{c}\text { Datos de } \\
\text { Identificación }\end{array}$ & $\begin{array}{c}\text { Datos de } \\
\text { Localización }\end{array}$ & $\begin{array}{l}\text { Jerar } \\
\text { quía }\end{array}$ & Descripción & Fotografía \\
\hline 1 & $\begin{array}{l}\text { Patrimonio: } \\
\text { Arquitectónico } \\
\text { Atractivo: } \\
\text { Iglesia San } \\
\text { Vicente }\end{array}$ & $\begin{array}{l}\text { Provincia: } \\
\text { Cotopaxi } \\
\text { Cantón: La } \\
\text { Maná } \\
\text { Parroquia: } \\
\text { La Maná } \\
\text { Coordenadas: } \\
\text { X: } 697574 \\
\text { Y: } 9895892\end{array}$ & I & $\begin{array}{l}\text { La religiosidad es un elemento } \\
\text { vivo en los lamanenses. Se } \\
\text { realizan misas semanales, } \\
\text { peregrinaciones, visitas a la iglesia } \\
\text { y conformación de grupos } \\
\text { juveniles de catequistas. Son } \\
\text { algunos elementos que aseguran la } \\
\text { vivencia de la religiosidad en La } \\
\text { Maná. } \\
\text { La iglesia fue construida hace más } \\
\text { de } 50 \text { años, ante la necesidad de un } \\
\text { lugar al cual acogerse los devotos } \\
\text { de San Vicente Ferrer. Dentro de } \\
\text { la iglesia está la imagen de este } \\
\text { santo, que fue traída al cantón en } \\
\text { el año de } 1970 \text { desde San Antonio } \\
\text { de Ibarra por el párroco Carlos } \\
\text { Jiménez en aquel entonces. }\end{array}$ & $\begin{array}{l}\text { Fuente: (Equipo de Trabajo, } \\
\text { 2019) }\end{array}$ \\
\hline
\end{tabular}

\section{Provincia:}

Patrimonio: Cotopaxi

Arquitectónico

Atractivo:

2 Iglesia del

Patrono San

Marcos

\section{Cantón: La}

Maná

Parroquia:

Guasaganda

Coordenadas:

X: 704761

Y: 9911116
A partir de construir el poblado, se establecen organizaciones y cooperativas campesinas en el año 1965, conjuntamente los primeros pobladores trabajaban y realizaban donaciones para la construcción de la iglesia San Marcos.
Figura 2: Iglesia del Patrono San Marcos

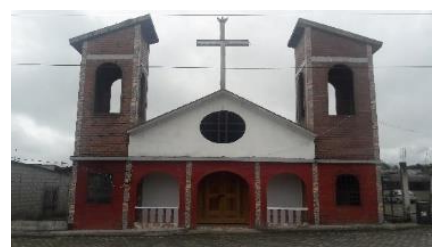

Fuente: (Olga Rosero, 2019)

Patrimonio:

Arquitectónico

3
Provincia:

Cotopaxi

Cantón: La

Iglesia del

Patrono San

Pablo
Maná

Parroquia:

Coordenadas:

X: 709176

Y: 9920612
Pucayacu es una parroquia del cantón La Maná en la provincia de Cotopaxi es el nombre con el cual sus pobladores le parroquializaron en las sesiones: ordinaria del 7 y extraordinaria del 27 de julio de 1949, siendo en ese momento los otros caseríos de Guadual y Choaló, parte de la naciente parroquia Pucayacu. El 29 de junio se festeja la fiesta de San Pablo de Pucayacu en honor al patrono de la parroquia (San Pablo). (Gabriela Armas, 2019)
Figura 3: Iglesia del Patrono San Pablo

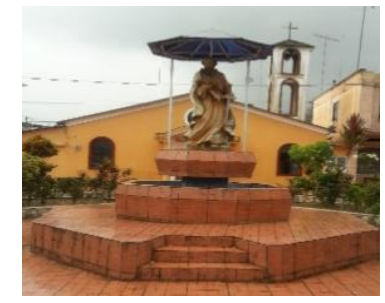

Fuente: (Gabriela Armas, 2019) 


\section{Provincia: \\ Cotopaxi \\ Cantón: La \\ Patrimonio: \\ Arquitectónico \\ 4 Atractivo: \\ Parque Lineal \\ "La Pista" \\ Maná \\ Parroquia: $\mathrm{La}$ \\ Maná \\ Coordenadas: \\ X: 697254 \\ Y: 9896077}

El proyecto del parque lineal es una obra civil implementada a partir de estudios topográficos para el aprovechamiento de una vía urbana ya existente, con una extensión aproximada de $1.115 \mathrm{~m}$ de longitud, donde se utilizó el espacio implementando facilidades y equipamiento para el desarrollo de actividades recreativas para niños, jóvenes, adultos, adultos mayores $\mathrm{y}$ adecuación de un área deportiva; la unión de estas actividades forma un conjunto destinado a la recreación y el deporte. (GAD La Mana, 2015)

La colección suma alrededor de 40 piezas clasificadas por Periodo, entre ellos: fragmentos de ollas ornitomorfas, cuencos fitomorfos, decorativos -zoomorfos-, platos con soporte anular, ollas con soporte trípode, compoteras, silbatos de figuras antropomorfas, entre otras.

Las investigaciones arqueológicas en la cuenca norte del Guayas realizada entre los años de 1992 al 2000 por el proyecto Valencia-Quevedo-La Maná, dieron como resultado la identificación de diferentes tipos de tolas, viviendas, áreas agrícolas, ceremoniales y basurales con asentamientos poblacionales del Período Formativo (Valdivia y Chorrera). (GAD La Mana, 2015)

La Manà - Av. Medardo Ángel Silva y Gonzalo Albarracín.

El Sr. Miguel Mullo Llego en el año 1988, actualmente tiene 30 años viviendo en el cantón, proviene de Pujilí, desde pequeño a la edad de 7 años ayuda hacer ataúd, sus padres eran bien rígidos y escultores, la profesión que ejerce es hereditaria de su papa, estudio en el colegio de artes plásticas "Daniel Reyes" de San Antonio de Ibarra, ha tenido dos aprendices, han mantenido la tradición también sus hermanos, sobrinos e hijos, son artistas en lo cual dibujan muy bien.

(Gabriela Armas, 2019).
Figura 4: Parque Lineal "La Pista"

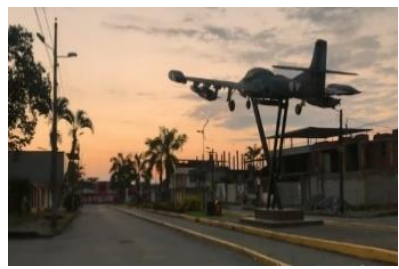

Fuente: (GAD. La Maná, 2015)

Figura 5: Museo

Arqueológico

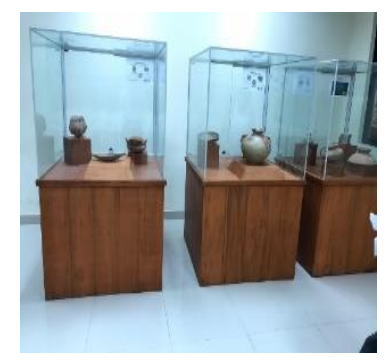

Fuente: (GAD. La Maná, 2015)

Figura 6: Tallado de figuras en mármol, resina de vidrio, bronce, madera $y$ restauraciones.

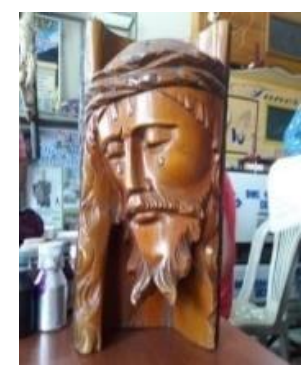

Fuente: (Gabriela Armas, 2019) 
ISSN: 2600-5859

En el cantón La Maná con el transcurso de los años se vinieron elaborando distintos tallados de artesanías aproximadamente desde hace 31 años, a partir de la ventaja de intuición se trabaja de manera artesanal. Sus inicios fueron con la conchiperla y el coral; años después los artesanos empezaron a realizar artesanías con la tagua o más conocida como mococha.
Figura 7: Artesanías en Tagua

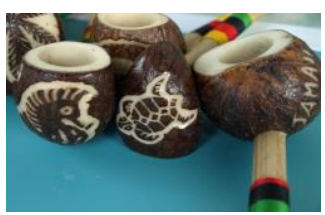

Fuente: (GAD. La Maná, 2015)
El grupo de artesanas está conformado por cinco mujeres que elaboran los trabajos con fibra de banano, entre sus productos se encuentran sombreros, bolsos, monederos, maseteros, recuerdos, bordado de botellas, entre otros; Patrimonio:

Cotopaxi

Artístico

Cantón: La

Atractivo:

Artesanías con

en Fibra de

Banano

Maná

Parroquia: El

Carmen

Coordenadas:

X: 697957

Y: 9898177
I

fueron capacitadas por el SECAP en el año 2010, el curso se desarrolló durante ocho días intensivos, en el cual aprendieron como procesar y trabajar las artesanías con la chonta del banano.
Figura 8: Artesanías con en Fibra de Banano

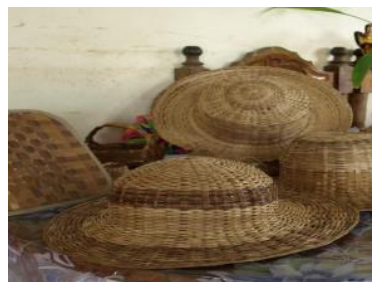

Fuente: (GAD. La Maná, 2015)

Figura 9: Libros de Oro de Guasaganda realizó el 10 de Enero de 1976 dándole por terminada la minga en ese mismo año, la sección de comité de fiesta de la parroquia de Guasaganda fue el 24 de Abril de 1977.

La duración de cuentas e informe de comisiones y cubierta de la capilla se dio en el año de 1983

Libro 2 Años De 1982 - 1991

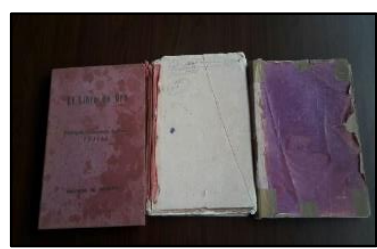

Fuente: (Gabriela Armas, 2017)

Ministerio de salud Srta. Janeth Ibáñez, Ministerio MAG Srta. Nery Calvopiña, Colegio Nacional y el agua.

Libro 3 Años De 1985 - 1999

DIRECTIVA FORMADA Y POSESIONADA POR EL ILUSTRE MUNICIPIO DE PUJILÍ (Gabriela Armas, 2019). 
En la Finca Agro Turística El Solaso se elaboran productos $100 \%$ naturales como: café, chocolate, mermelada de guayaba, harina de plátano, licor de café, licor de cacao; la materia prima es transformada mediante una pequeña procesadora artesanal, el registro sanitario de estos productos se encuentran en trámite.

Industrial

ncia:

Cantón: La

10

Atractivo:

Maná

Finca

Orgánica "El

Solaso"

Parroquia: El

Triunfo

Coordenadas:

X: 692197

Y; 9895745

\section{I}

Para realizar el chocolate él lo cosecha y le saca las pepas y lo pone a secar por 15 días ya teniendo seca las pepas le pone a tostar en una maquina tostadora de leña, lo pone 25 libras de cacao por hora, una vez tostado se trilla se lo pela el cacao y el último proceso es el molino, y obtenemos el chocolate puro.

Procesadora de Lácteos 3K" realiza dentro de su establecimiento el proceso del yogurt y queso el cual tiene cuatro etapas y son: Coagulación, Drenaje o desuerado, Prensado. Proceso del yogurt: Se utiliza la leche dependiendo la cantidad que se va realizar en unos 100 litros se le agrega azúcar 4 kilos, se mide la leche que este a $75^{\circ}$ y después a los $90^{\circ}$ después de todo el proceso toca dejar enfriar y meciéndole para que no se asiente cuando ya estece en $40^{\circ}$ ya se le coloca el fermento del yogurt, se tapa y se manda a entubar por 8 horas, una vez eso se le pone persevantes y los sabores artificiales.

Varios acontecimientos ha marcado la historia del recinto Malqui desde la llegada de los terratenientes a suelos del recinto, sobre la explotación laboral a los habitantes y a la llagada de algunas congregaciones. Casos que de alguna $\mathrm{u}$ otra manera ha contribuido a la situación actual de cientos de familias que en su mayoría se caracterizan por ser un pueblo unido.

Los primeros habitantes del recinto Malqui llegaron desde diferentes partes de la provincia, trabajaban para el terrateniente cuidando sus animales y tierras, a
Figura 10: Finca Orgánica

"El Solaso"

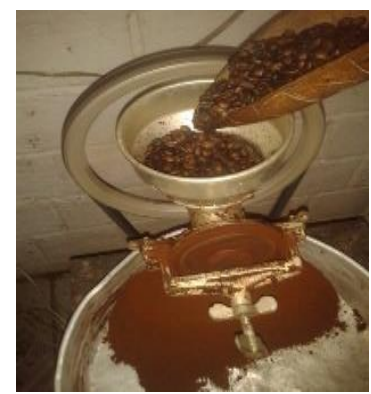

Fuente: (Sr. Eduardo Lara, 2018)

Figura 11: Lácteos 3K

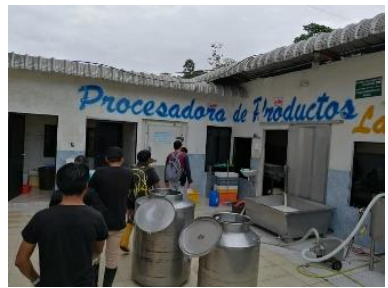

Fuente: (Cristian Espinosa, 2019)

Figura 12: Molienda

Tradicional de Caña

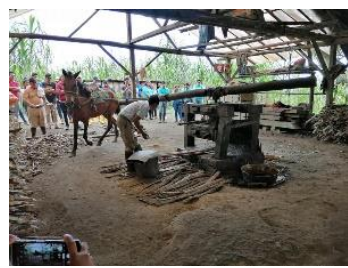


ISSN: 2600-5859

cambio solo recibían una pequeña parcela de tierra para poder sobrevivir sembrando sus alimentos ya que en aquellos tiempos no tenían un salario, mediantes estas pequeñas parcelas que fueron dadas por el terrateniente, los habitantes optaron por la construcción de trapiches, en la actualidad en el recinto Malqui de la parroquia de Guasaganda del Cantón La Maná, se encuentra alrededor de 7 trapiches, 2 artesanales y 5 modernizados. (Gabriela Armas, 2019).

Se lo considera como un sitio místico de los Andes que ha sido venerado como uno de los lugares más sagrados de las civilizaciones antiguas; en el sector conocido como Estero Hondo el Ing. Guillermo Sotomayor (científico) descubrió que el agua que brotaba al pie de la ladera del sitio conocido como la Montaña Sagrada de La Maná, tenía oro; las muestras de esta agua han sido analizadas por catorce laboratorios de los EE.UU. y por el Instituto de las Bebidas Austriaco, además de pruebas caseras. En este sector también se han realizado importantes hallazgos arqueológicos identificándose características propias de la cultura sumeria.

(GAD La Mana, 2015)
Fuente: (Cristian Espinoza, 2019)
Figura 13: Envasadora de Agua Esplendor

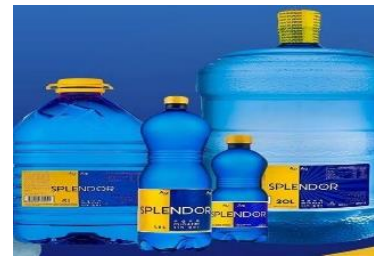

Fuente: (Agua Esplendor, 2015)
Según hipótesis de la historiadora Tamara Estupiñán $(2015$, p. $27-$ 29), la construcción data de la época del pueblo Inca, "Machay significa gruta, santuario en el que se encuentra el Malqui. El sitio que alberga las ruinas de Machay pertenece a la región de Malqui, y Malqui significa cuerpo o momia de los ancestros del Inca.
Figura 14: Ruinas de Machay.

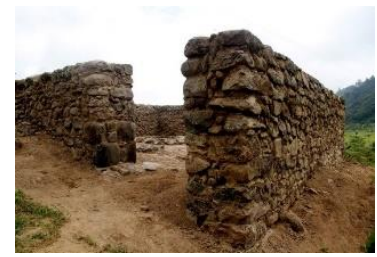

Fuente: (Proyecto Machay, 2015) 


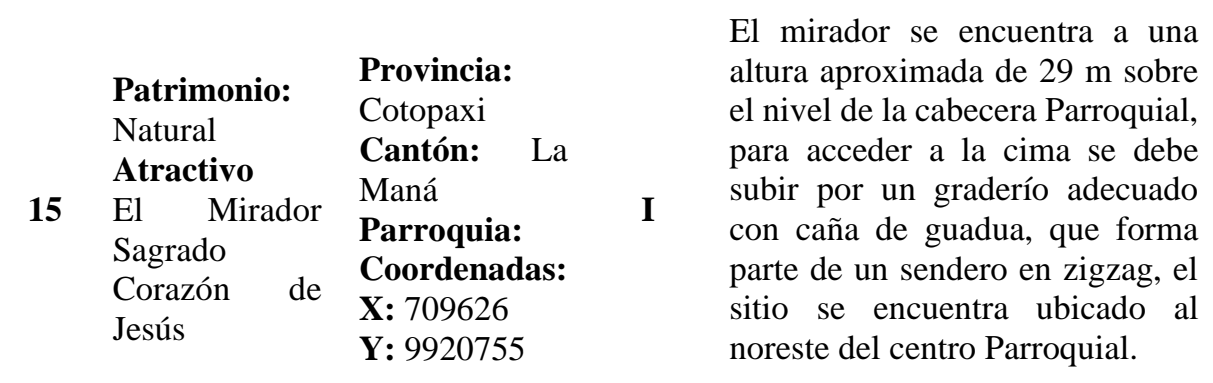

Figura 15: El Mirador Sagrado Corazón de Jesús

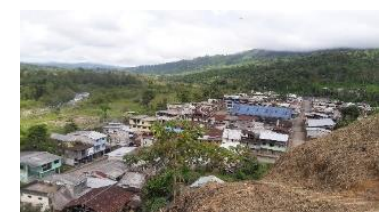

Fuente: (Olga Rosero, 2018).

\section{Discusión}

Según el PDOT (2015, p. 15) "El cantón La Maná está localizado en las estribaciones de la cordillera occidental de Los Andes, en la provincia de Cotopaxi, a 150Km de Latacunga capital de la provincia. Morfológicamente se ubica sobre una llanura de pie de cordillera compuesta de depósitos aluviales cubiertas de cenizas y arenas volcánicas de origen desconocido. La cabecera Cantonal se asienta sobre una terraza aluvial antigua del río San Pablo. Tiene varios pisos climáticos que varía de subtropical a tropical (altura variable de 200 y 1150 msnm).”

El cantón La Maná es uno de los cantones primordiales en riqueza cultural y natural de la provincia de Cotopaxi. Dentro del cantón la información de los atractivos turístico culturales es escasa y con este proyecto se pretende resolver la problemática, la falta de información y mantenimiento de los mismo, con ello también se aportara como vision para el turismo comunitario del cantón. Se trabajara en el diagnotico del patrimonio cultural tangible para aportar al valor cultural que posee la comunidad.

El patrimonio cutural esta contituido por bienes muebles e inmuebles, donde se puede identificar a la categoria que pertenecen, ya sea: Monumentos.Manuscritos, Grabaciones, Artefactos,Peliculas,Fotografias,Pinturas, entre otros.

Se trata de enfocar en un turismo comunitario, ya que hoy en dia los turistas buscan que sus actividades sean vivenciales y ante esto conocemos que el turimo se alimenta de las investigaciones realizadas por las personas que se guían en el tema cultural, como se lo pretende hacer mediante esta investigacion, la finalidad del mismo es de darle un punto de vista turistico cultural a la comunidad para que mediante sus atractivos culturales aporten al desarrollo economico de la localidad y asi mejorar su calidad de vida. 
Como parte del proyecto también es dar a conocer a la ciudadanía involucrada en los atractivos culturales del cantón, mediante la gestión que implica realizar un diagnóstico del Patrimonio Cultural Tangible que posee el cantón para que vaya en crecimiento la demanda turística y se mejore la calidad de servicios turisticos que ofrencen los establecimiento y asi contribuya al desarrollo turistico comunitario del cantón La Maná.

\section{Conclusiones}

- El inventario de los sitios culturales tangibles del cantón La Maná se encuentra creado por: bienes inmuebles, y manifestaciones religiosas que al juntarse dan un total de quince bienes los mismos que son de tipo artesanal y religiosos estos bienes culturales se encuentran en buenas condiciones físicas para ser promocionados como atractivos turísticos.

- Para conservar un bien tangible cultural se debe valorizar las condiciones arquitectónicas o culturales que lo conllevaron a convertirse en un sitio cultural, de esta manera el bien puede ser de gran beneficio para la sociedad para así poder respaldar su permanencia y salvaguarda cada uno de los lugares culturales que tiene el cantón.

- Mediante el diagnostico situacional se pudo evidenciar que el patrimonio cultural tangible dentro del cantón, no tiene un incremento sustancial, sin embargo, los sitios culturales carecen de mantenimiento y promoción lo que ha ocasionado que el sector no se desarrolle turísticamente por esta razón se pudo determinar que el diagnóstico del patrimonio cultural incide de una u otra forma el desarrollo turístico y económico del sector.

\section{Referencias bibliográficas}

Caiza , K., \& Mora, V. (2016). La Maná Tierra de Encantos (Vol. 1). La Maná. Recuperado el 24 de Abril de 2020

Chasiluisa, R., \& Cupuerán, M. (2012). Plan De Desarrollo Turístico Comunitario Para La Parroquia De Tumbabiro, Cantón Urcuqui, Provincia De Imbabura. Imbabura. Recuperado el 24 de Abril de 2020, de http://www.dspace.uce.edu.ec/bitstream/25000/885/1/T-UCE-0005-148.pdf

El Instituto Nacional de Estadística y Censos. (2010). Población del cantón La Maná. 2. Recuperado el 21 de Febrero de 2020, de https://www.ecuadorencifras.gob.ec/documentos/webinec/Bibliotecas/Fasciculos_Censales/Fasc_Cantonales/Cotopaxi/Fasciculo_La_Mana. pdf 
García, J. A., RedingBernal, A., \& LópezAlvarenga, J. C. (2013). Cálculo del tamaño de la muestra en investigación. 6. Recuperado el 21 de Febrero de 2020, de http://www.scielo.org.mx/pdf/iem/v2n8/v2n8a7.pdf

INPC. (2011). Instructivo para Fichas de Registro e Inventario, Patrimonio Cultural Inmaterial. Recuperado el 24 de Abril de 2020, de https://downloads.arqueoecuatoriana.ec/ayhpwxgv/noticias/publicaciones/INPC-X-

InstructivoParaFichasDeRegistroInventarioPatrimonioInmaterial.pdf

La Maná Noticias. (11 de Diciembre de 2017). Proyecto Machay. Recuperado el 24 de Abril de 20202, de https://lamanoticias.wordpress.com/2017/12/11/malqui-machay-refugiodel-ultimo-rey-inca/

La Organización de las Naciones Unidas para la Educación, la Ciencia y la Cultura. (2009). 20 Recuperado el 21 de Febrero de 2020, de https://unesdoc.unesco.org/ark:/48223/pf0000187828

MINISTERIO DE CULTURA Y PATRIMONIO. (s/f). MINISTERIO DE CULTURA Y PATRIMONIO. Recuperado el 24 de Abril de 2020, de https://www.culturaypatrimonio.gob.ec/en-cotopaxi-la-interculturalidad-une-a-lascomunas/

Peña, J. M. (2013). El patrimonio cultural, bibliográfico y documental de la humanidad. Revisiones conceptuales, legislativas e informativas para una educación sobre patrimonio. Cuicuilco, 32.

Popovski, P., Trillingsgaard, Kasper, Simeone, O., \& Durisi, G. (2018). 5G Wireless Network Slicing for eMBB, URLLC, and mMTC: A Communication-Theoretic View. Open Access Journal, 6. 
PARA CITAR EL ARTÍCULO INDEXADO.

Rosero Perez , O. S., Yepez Arauz, N. S., Armas Cedeño, G. I., \& Zambrano Cuadro, N. G. (2020). Diagnóstico del patrimonio cultural tangible como visión para el turismo comunitario en el cantón La Maná Provincia de Cotopaxi - Ecuador. ConcienciaDigital, 3(3.1), 6-16. https://doi.org/10.33262/concienciadigital.v3i3.1.1346

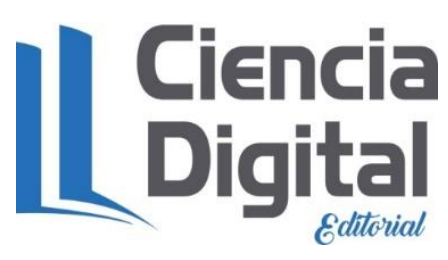

El artículo que se publica es de exclusiva responsabilidad de los autores y no necesariamente reflejan el pensamiento de la Revista Explorador Digital.

El artículo queda en propiedad de la revista y, por tanto, su publicación parcial y/o total en otro medio tiene que ser autorizado por el director de la Revista Explorador Digital.
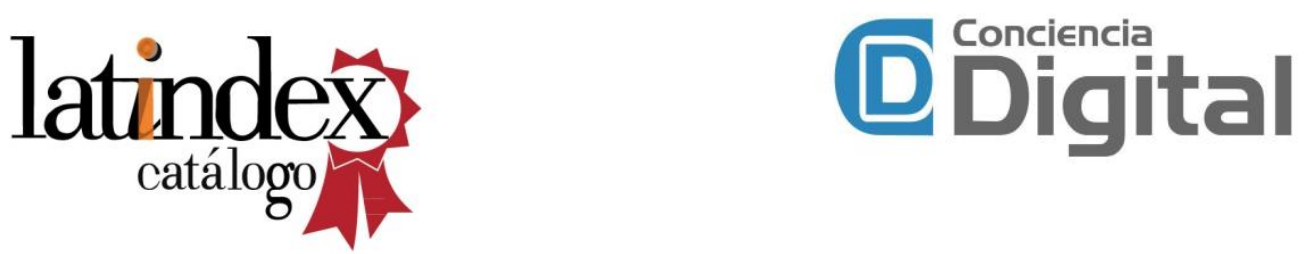